\title{
A pandemic associated with novel coronavirus of probable animal origin
}

\author{
Navroop Kaur ${ }^{1 *}$, Saurabh Kumar Jha ${ }^{2}$, Premnidhi Yadav ${ }^{3}$, Soni Mishra ${ }^{3}$, Umar Farooq $^{4}$, Umme Salma Siddiqui ${ }^{5}$, \\ Mohammad Zuhaib ${ }^{6}$, Arshi Amin ${ }^{7}$, Niraj Kumar Jha ${ }^{8}$, Ankur Sharma ${ }^{9}$
}

\author{
${ }^{1}$ Assistant Professor, Department of Microbiology and Biotechnology, Noida International University, Gautam Budh \\ Nagar-201308, Greater Noida, Uttar Pradesh, INDIA \\ ${ }^{2}$ Assistant Professor, Department of Biotechnology, Sharda University, Greater Noida, Uttar Pradesh, INDIA \\ ${ }^{3}$ Noida International University, Gautam Budh Nagar, Uttar Pradesh, INDIA \\ ${ }^{4}$ Associate Professor Microbiology, Faculty of Dentistry, Taif University, Saudi Arabia \\ ${ }^{5}$ Department of Chemistry, Brooklyn College \& the graduate center, City University of New York, USA \\ ${ }^{6} \mathrm{Head}$ of Department, Department of Agriculture, Noida International University, Uttar Pradesh, INDIA \\ ${ }^{7}$ Assistant professor, Department of Chemistry, Noida International University, Uttar Pradesh, INDIA \\ ${ }^{8}$ Assistant professor, Department of Biotechnology, Sharda University, Greater Noida, Uttar Pradesh, INDIA \\ ${ }^{9}$ Assistant professor, Department of Life Science, Sharda University, Greater Noida, Uttar Pradesh, INDIA
}

Received: 08 May, 2020

Accepted: 19 June, 2020

*Correspondence to:

Dr. Navroop Kaur,

Email:

navroop.saggu9@gmail.com

Copyright: (C) the author(s), publisher and licensee Indian Academy of Pharmacists. This is an open-access article distributed under the terms of the Creative Commons Attribution NonCommercial License, which permits unrestricted noncommercial use, distribution, and reproduction in any medium, provided the original work is properly cited.

Published by: OZZIE Publishers

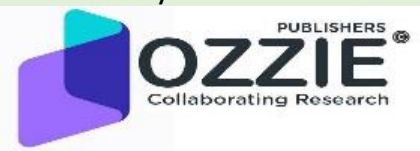

\section{Abstract}

Emerging infectious diseases, such as (SARS) Severe Acute Respiratory Syndrome coronavirus and (MERS) Middle East Respiratory Syndrome coronavirus, it is the third extremely pathogenic coronavirus which is emerged in the last two decades. Epidemiological investigation recommended that coronavirus outbreak was associated with seafood marked in Wuhan, China. The patient suffering from coronavirus experience various respiratory syndrome symptoms including temperature, faintness and cough. Since, the cause of coronavirus was unknown. The analysis of pneumonia was unidentified, and pneumonia was identifying depending on clinical characteristics, ruling out of common bacterial, viral pathogens and chest imaging that causes pneumonia. Some more cases were confirmed in China, Japan, USA and India. In todays, Wuhan, China there is possibility of SARS-CoV outbreak without any earlier notice. To prevent this epidemic various case from different countries should implement better triage schemes for imported cases of SARS -CoV. Some precautions like N95 masks were used for aerosol generating diseases. One of the experimental antiviral agent Remdesivir drug shows significant activity against coronavirus in vitro. There is no exact treatment for this virus infection, however, can relieve symptoms by taking over counter medicine for pain, fever and cough, using a room humidifier. As there is no exact treatments are available for this virus, initial control and prevention of more spread will be critical to halt the outbreak and to control the spread of this coronavirus infections.

KEYWORDS: Novel Coronavirus (COVID-19), pandemic, anti-viral drugs, treatment.

\section{INTRODUCTION}

An outbreak of (SARS-CoV) severe acute respiratory syndrome-related coronaviruses have been revealed firstly in natural reservoir i.e., host and bats [1-4]. Coronavirus belongs to the family of viruses that causes sore throat to more severe respiratory infections, or SARS and can lead to death. Coronavirus is a major danger to public health and is broadly distributed in the humans. The virus spread through person-to-person both in hospital and family $[5,6]$. Coronaviruses can be transmitted from contaminated surfaces and it is mainly transmitted through mucous membranes of the eyes, nose or mouth [7, 8], highlighting the significance of coronavirus persistence on inanimate surfaces [9]. The recent cases appeared in the Chinese city (Wuhan) in December 2019. At the South China Agricultural University, two researchers in Guangzhou have proposed that pangolins -these are long-snouted mammals that are frequently used in Chinese medicine - are used as the animal source of the coronavirus outbreak. These mammals may be the possible cause of the virus, named as COVID-19 (2019 novel coronavirus), based on a genetic comparison 
towards coronaviruses taken from the animals and humans infected in the outbreak. Scientists have proposed that may be COVID-19 originally came from bats, based on the similarity of the genetic sequence to known coronaviruses, but the virus was probably transmitted from animals to humans.

More than above 1200 people in China have died from COVID-19, a larger number than those individuals who died in 2002-03 from SARS epidemic, which is also originated in China, killing of 775 individuals all over the world. World Health Organization (WHO) has stated the COVID-19 epidemic a global health emergency in March 2020. The worldwide impact of this new epidemic is yet undefined. There is no defined treatment for coronavirus, but we can treat the symptoms. So, in this review some of its treatment goals and prevention methods are discussed.

\section{Epidemiological studies of coronavirus:} Coronaviruses are incredibly diverse and are zoonotic in nature. It is commonly come across during the flu and winter season in clinical practice. Numerous types of biocides such as hydrogen peroxide, alcohols, sodium hypochlorite and others are used globally for sterilization, mainly in healthcare areas [10]. Moreover, there are no specific treatments for this COVID-19. The common names of COVID-19 are coronavirus HKU1, NL63, 229E and OC43. In this review, we will go through the epidemiological studies of coronavirus.

Two another coronaviruses, SARS-CoV (Severe acute respiratory syndrome) or are emerged in 2003, and MERS-CoV (Middle east respiratory syndrome) are emerged in 2012, which have been shown to infect humans. These two viruses are linked with very high death rates. Now a days, COVID-19, has been occurred in Wuhan in the year 2019. There are more than thousands of cases in China and numerous cases across the worldwide including Hong Kong, Macau, Japan, USA, South Korea and India etc.

\section{Classification of coronavirus}

It is a pathogen, called beta-coronavirus, which is very much alike to SARS (severe acute respiratory syndrome) and MERS (Middle East respiratory syndrome). [2]. Coronaviruses having enveloped positive sense 3 RNA viruses which ranges from $60 \mathrm{~nm}$ to $140 \mathrm{~nm}$ in diameter also having spikes on its surface, which can be seen under the electron microscope [11]. There are mainly four corona viruses namely HKU1, NL63, 229E and OC43 have been in early identified in humans and those cause mild respiratory infections.

\section{Clinical manifestation of coronavirus}

It is associated with respiratory tract infection which supposed to be a zoonotic virus that has been mutated that permit human pathogenicity [1]. The infection varies from asymptomatic to severe in patients with clinically evident infection [1]. $2 \%$ is the mortality rate of the identified cases and rest is unfinished and growing. Furthermore, COVID-19 is a mutated and recombines every so often, which shows a challenge to the human understanding and its clinical organization. Patients with coronavirus infection may develop after the completion of course, within a week or longer and starting with minor indications, it progresses to severe symptoms like shock and dyspnea. Most common symptoms of COVID-19 are sore throat, cough, myalgia and fatigue [3, 4]. Some patients complain about of dyspnea with moderate to severe symptoms. Hemoptysi and pleuritic chest pain has also been reported in some patients [12]. Some of the UTI infections, headache, cough, sneezing, sore throat, nausea, diarrhea and vomiting has also been reported [3]. Patients may get infection of coronavirus from nearby contact with an COVID-19 infected person and an epidemic area depending upon its travel history (within 14 days) and the area already infected with the same infection [13]. Patients with coronavirus infection may give the impression of moderately infected with tachypnea and fever, which having $39^{\circ} \mathrm{C}$ of temperature. Patients with the age of 60 and those are immunodeficient may not have fever but are prone to coronavirus [3].

\section{Causes and risk factors}

It is documented that COVID-2019 infection transmitted through person-to-person and is assumed to occur by close contact with infected person certainly via respiratory droplets $[12,13,14]$. Coronavirus is not yet recognized within its development stage of infection, when the person can be transmissible to other person. Some scientists reported that coronavirus may be communicated before the development of symptoms. An incident reported from Germany has been already published but is uncertain, the frequency of spread is not yet known $[15,16]$.

Risk factors are associated with some factors. 1) Age: Most of the reports shows that cases belongs adults of middle age (30-60) and older than age of 60 have been infected with the coronavirus. 2) Gender: In published cases, in comparison to females, males have been affected more. Additional risk factors are associated with diseased persons and seafood market, in Wuhan. The infection was expected to be developed when a person exposed to diseased animals also [17]. Though the environmental samples which are associated with infected marketplace revealed the confirmation of coronavirus, but there is no animal samples give positive results, therefore, the original source of coronavirus are still mysterious [18]. 


\section{Diagnostic procedure for coronavirus}

Coronavirus supposed to infect those individuals who might have affinity to exposure history and respiratory infection. Through chest imaging, it is documented that the existence of pneumonia and also assesses severity, by plain radiography and $\mathrm{CT}$ scan has been used for the detection. Oxygenation would be measured by two tests i.e. pulse oximetry or by arterial blood gas test. After that a PCR test has been done. The culturing of coronavirus is not suggested. WHO and CDC have considerably not the same criteria for whom to test or not. Those having travel history within 14 days can be tested for infection [13]. If occurrence of this infection increases in other areas, the criterion may expand. There are various countries reporting just a single case to multiple of cases. $\mathrm{WHO}$ and CDC defines that direct contact within $2 \mathrm{~m}$ i.e. $6 \mathrm{ft}$ or within a single room or within hospital or hospital area or person without using any safeguarded or protective equipment or close contact with eyes, nose, hands secretions person having COVID-19 infection. Any healthy person can get infection. Exposure to the person who is associated with COVID-2019 infections has also been reported. Some doctors, nurses, health care workers and cleanliness workers, person having travel history, these infected patients with coronavirus may infect the normal persons working in hospitals. Some other physical symptoms may shows the signs of COVID-19 like dullness, faint, slower breath sounds etc. Through some clinical examination, people infected with COVID-19 may be found like chest radiography shows diffuse patchy infiltrates. Chest radiography tests shows results like lobar consolidation or localized patchy infiltrate which helps in finding the COVID-19 virus. With the help of sputum examination, it can detect abundant polymorph nuclear leukocytes and legionella antigens might be detected in urine, sputum culture, through which the coronavirus infection can be diagnosed.

\section{Treatment goals for corona virus}

Recently, an antiviral drug namely Remdesivir was stated as a hopeful drug against wide selection of RNA viruses. Holshueet al. stated that people infected with COVID-19, having treated with remdesivir antiviral drug resulted in achieving good results [19, 20]. After that, Xiao et al. findings disclose about the remdesivir antiviral drug is efficiently works on the control of COVID-19 in vitro studies. [21]. For the time being, some scientists also found that there is a drug namely chloroquine having an immune-modulating activity, previously which is used to treat malaria. It's a synthetic form of quinine. Quinine is a natural compound, which is found in the bark of cinchona tress. Chloroquine works by principally slow down the virus entry to cells, which can slow down the rate of replication. Chloroquine could efficiently inhibit this virus in lab conditions also. [22]. This drug was proved to be effective for the treatment of patients having infection with coronavirus in clinical trials [23]. Clinical trials of another drug namely remdesivir is also undergoing, but the final efficiency of this drug is still unclear. An another indole derivative molecule, which is small in size called as Arbidol, was found to block the virus-related combination against influenza $\mathrm{A}$, influenza $\mathrm{B}$, hepatitis $C$ virus [24] and also having antiviral effect against SARS-CoV in vitro [25]. It may be potent as a drug in future against COVID- 19 treatment. Another drug namely, Kaletra is under study, which is a combination of two antiviral drugs, one is lopinavir and another one is ritonavir, which are used to fight against HIV infection. It is commonly available and its clinical trials are ongoing. Both the drugs complement each other. Lopinavir drug stops viral enzymes from cutting up main proteins that are significant to HIVs reproduction. Ritonavir helps in enhancement of lopinavir's concentrations in cells. But a recent study suggests that arbidol drug has much improved therapeutic effect than kaletra, which considerably decrease the rate of infection in various cases. Furthermore, lopinavir/ritonavir, nucleoside analogues, neuraminidase inhibitors, peptide EK1, remdesivir may be used as antiviral drugs against COVID-19 treatment [26]. On the hand, Chinese herbal drugs like astragalus, baizhu, fangfeng and honeysuckle which are mostly used to treat other infections. But there decoction is not appropriate for its long-term use, and one week is its finest period [27]. Some studies elaborate that lower respiratory tract infections can be cured by vitamin $\mathrm{C}$ under certain conditions [28], Consequently, an adequate quantity of vitamin $C$ supplementation might be an approach in the treatment of COVID-2019. CDC provides guidance to implement the medical care approvals and infection controller measurements aimed for people [29]. WHO also offers measures for the treatment and prevention of severe pneumonia [15]. At present, there is no definite antiviral drug which is accepted for the treatment of COVID-19. But the research for the novel drug is still going on. The clinical trials and protocols based on in vitro activity are being used on numerous existing antiviral agents. FDA-approved the lopinavir-ritonavir drugs for the treatment of HIV. It is also used for SARS and for the treatment of MERS also [31, 32]. In- vitro, Remdesivir, antiviral agent significantly showed activity against coronaviruses $[33,30]$. There is no exact treatment for coronavirus, but the symptoms can be treated.

\section{CONCLUSION}

In conclusion, coronavirus is a serious infectious disease, and then SARS-CoV-2 and the chief indications are fever, tiredness, dry cough and fatigue, that are alike to SARS. Corona viruses are a huge family of viruses which may be the source of infections in human's beings. Individuals having respiratory tract infections that ranges from the cold to severe diseases and even death. Even though, it spreads from human-to-human and it is 
confirmed. Some patients may have symptoms like nose congestion, sore throat, fever and diarrhea etc. These indications are usually minor and increase gradually. Some individuals become contaminated by COVID-19 but they don't change symptoms and even don't feel ill. Older people above age 60, and those having medical problems like high blood pressure, heart problems, hypertension, heart disease and diabetes, lung disease, kidney disease, patients on cancer medications are more likely to develop severe infection are more susceptible to this virus because they have very low immunity in the body [34]. As a result, it is mainly significant to enhance the self-resistance of a person. The significant method to enhance one's immunity is to maintain the hygiene at personal level, a good life and suitable nutritive intake is one of the best techniques $[35,36]$. Those persons, taking defensive methods like wearing medical masks, adequate rest, creating supportive environment, home quarantine self, home quarantine family members, and home care can be proficiently helps in avoiding COVID19 [37]. The WHO mentions "ensuring that cleaning of environment, stop polluting the environment and disinfection measures must be followed correctly. Methodically cleaning of all surfaces of objects with water and detergent and then applying different disinfectants like sodium hypochlorite are best methods." [38]. Various medications like ribavirin, interferon, lopinavir-ritonavir, corticosteroids have been used on patients infected with different viruses like SARS or MERS, though the effectiveness of some medications remains doubtful. Chloroquine was proved to be effective for the treatment of patients having infection with COVID- 2019 in vitro [23]. Favilavir anti-viral drug may be used for the cure of COVID-19 infection. This drug has been shown efficiency in the treatment of disease with lesser side effects in vitro. The spreading of COVID-19 in hospital area can be successfully prohibited when appropriate procedures are accomplished [39, 40]. There is no exact treatment for coronavirus, but the symptoms can be treated.

\section{List of Abbreviations}

SARS-Severe Acute Respiratory Syndrome coronavirus MERS- Middle East Respiratory Syndrome

WHO- World health organization

SARS-CoV- Severe acute respiratory syndrome-related coronaviruses

COVID-2019- Novel coronavirus 2019

CDC-Centers for disease control

ICU- Intensive care unit

CT scan- Computed tomography scans

PCR- Polymerase chain reaction

$\%$ - Percentage

Eg.- For Example

nm-nanometer

m-meters

$\mathrm{ft}-\mathrm{feet}$

\section{CONFLICT OF INTEREST}

The authors declare that they have no conflicting interests.

\section{ACKNOWLEDGEMENT}

The assistance provided by Dr. Saurabh kumar Jha was greatly appreciated. I would like to thank all the authors for helping me in finalizing this paper.

\section{REFERENCES}

1. W H O. Novel Coronavirus (2019-nCoV): Situation Report-13, W H O website. Published February (2) 2020.

2. Zhu N.A novel coronavirus from patients with pneumonia in China 2019.N Engl J M ed. ePub, 2020.

3. Huang C. Clinical features of patients infected with the 2019 novel coronavirus in Wuhan, China. Lancet.ePub, 2020.

4. Chen N. Epidemiological and clinical characteristics of 99 cases of 2019 novel coronavirus pneumonia in Wuhan, China: a descriptive study, Lancet. ePub, 2020.

5. De Wit E, van Doremalen N, Falzarano D, Munster VJ. SARS and MERS: recent insights into emerging coronaviruses. Nat Rev Microbiol 2016;14: 523e34.

6. Chan JF, Yuan S, Kok KH, To KK, Chu H, Yang J. A familial cluster of pneumonia associated with the 2019 novel coronavirus indicating person-to-person transmission: a study of a family cluster. Lancet 2020; 6736(20) : 30154-9.

7. Otter JA, Donskey C, Yezli S, Douthwaite S, Goldenberg SD, Weber DJ. Transmission of SARS and MERS coronaviruses and influenza virus in healthcare settings: the possible role of dry surface contamination. J Hosp Infect 2016; 92:235e50.

8. Dowell SF, Simmerman JM, Erdman DD, Wu JS, Chaovavanich A, Javadi M. Severe acute respiratory syndrome coronavirus on hospital surfaces. Clin Infect Dis 2004; 39:652e7.

9. Geller C, Varbanov M, Duval RE. Human coronaviruses: insights into environmental resistance and its influence on the development of new antiseptic strategies. Viruses 2012; 4:3044e68.

10.Kampf G. Antiseptic stewardship: biocide resistance and clinical implications. Cham: Springer International Publishing, 2018.

11.Richman DD, Whitley RJ, Hayden FG. Clinical Virology, 4th ed. Washington: ASM Press, 2016.

12.Chan J.F.W. A familial cluster of pneumonia associated with the 2019 novel coronavirus indicating person-to-person transmission: a study of a family cluster, Lancet. E Pub, 2020

13.CDC. 2019 Novel Coronavirus: Interim Guidance for Healthcare Professionals. CDC website. February 3, 2020.

14.CDC. 2019 Novel Coronavirus: How (2019-nCoV) Spreads. CD C website. February 3, 2020. 
15.WHO. Novel Coronavirus (2019-nCoV): Situation Report-7. WHO website. Published January 27, 2020.

16. Rothe C. Transmission of 2019-nCoV infection from an asymptomatic contact in Germany (letter to the editor; a counterargument reply is in press). $\mathrm{N}$ Engl $\mathrm{J}$ Med. ePub, 2020.

17. WHO. Disease Outbreak News: Pneumonia of Unknown Cause--China. WHO website. Published February 3, 2020.

18.China CD C. Notes From the Field: An Outbreak of N CIP (2019-nCoV) Infection in China-Wuhan, Hubei Province, 2019-2020. China CD C. 2(5):79-80, 2020.

19.Holshue ML, DeBolt C, Lindquist S, Lofy KH, Wiesman J, Bruce H. First Case of 2019 Novel Coronavirus in the United States.The New England journal of medicine, 2020.

20.Holshue ML, DeBolt C, Lindquist S, Lofy KH, Wiesman J, Bruce H. First Case of 2019 Novel Coronavirus in the United States. The New England journal of medicine, 2020.

21.Xiao F, Tang M, Zheng X, Li C, He J, Hong Z. Evidence for gastrointestinal infection of SARSCoV-2. medRxiv. 2020:2020.02.17.20023721.

22.Wang M, Cao R, Zhang L, Yang X, Liu J, Xu M. Remdesivir and chloroquine effectively inhibit the recently emerged novel coronavirus $(2019-\mathrm{nCoV})$ in vitro. Cell Res, 2020.

23.Gao J, Tian Z, Yang X. Breakthrough: Chloroquine phosphate has shown apparent efficacy in treatment of COVID-19 associated pneumonia in clinical studies. Biosci Trends, 2020.

24.Boriskin YS, Leneva IA, Pecheur EI, Polyak SJ.Arbidol: a broad-spectrum antiviral compound that blocks viral fusion. Curr Med Chem. 2008;15: 997-1005.

25.Khamitov RA, Loginova S, Shchukina VN, Borisevich SV, Maksimov VA, Shuster AM. [Antiviral activity of arbidol and its derivatives against the pathogen of severe acute respiratory syndrome in the cell cultures].VoprVirusol. 2008; 53: 9-13.

26.Lu H. Drug treatment options for the 2019-new coronavirus (2019-nCoV). Biosci. Trends. 2020.

27.Luo H, Tang QL, Shang YX, Liang SB, Yang M, Robinson N. Can Chinese Medicine Be Used for Prevention of Corona Virus Disease 2019 (COVID19)? A Review of Historical Classics, Research Evidence and Current Prevention Programs. Chin J Integr. Med, 2020.

28.Hemila H. Vitamin C intake and susceptibility to pneumonia. Pediatr Infect Dis J.1997;16: 836-7.
29.CDC. 2019 Novel Coronavirus: Interim Guidance for Implementing Home Care of People Not Requiring Hospitalization for 2019 Novel Coronavirus (2019nCoV). CD C website. Accessed February 3, 2020.

30. Sheahan TP. Comparative therapeutic efficacy of remdesivir and combination lopinavir, ritonavir, and interferon beta against MERS-CoV. $\mathrm{N}$ at Commun. 2020;11(1): 222.

31.ChuCM. Role of lopinavir/ritonavir in the treatment of SARS: initial virological and clinical findings. Thorax 2004; 59(3): 252-6.

32.Arabi YM. Treatment of Middle East respiratory syndrome with a combination of lopinavir-ritonavir and interferon- $\beta 1 \mathrm{~b}$ (MIRACLE trial): study protocol for a randomized controlled trial. Trials 2018;19 (1): 81.

33.Agostini ML. Coronavirus susceptibility to the antiviral remdesivir (G S-5734) is mediated by the viral polymerase and the proofreading exoribonuclease. mBio. 2018; 9(2).

34.Liang W, Guan W, Chen R, Wang W, Li J, Xu K. Cancer patients in SARS-CoV-2infection: a nationwide analysis in China. Lancet Oncol. 2020.

35.High KP. Nutritional strategies to boost immunity and prevent infection in elderly individuals.Clin Infect Dis 2001; 33: 1892-900.

36.Simpson RJ, Kunz H, Agha N. Graff R. Exercise and the Regulation of Immune Functions. ProgMolBiolTranslSci 2015;135: 355-80.

37.Guan Wj, Ni Zy, Hu Y, Liang Wh, Ou C-q, He Jx. Clinical characteristics of 2019 novel coronavirus infection in China, 2020

38. WHO. Infection prevention and control during health care whennovel coronavirus ( $\mathrm{nCoV}$ ) infection is suspected. WHO; 2020. Interim guidance. 25 January 2020.

39.Wiboonchutikul S, Manosuthi W, Likanonsakul S, Sangsajja C,Kongsanan P, Nitiyanontakij R. Lack of transmission amonghealthcare workers in contact with a case of Middle East respiratorysyndrome coronavirus infection in Thailand. AntimicrobResist Infect Control 2016; 5:21.

40. Ki HK, Han SK, Son JS, Park SO. Risk of transmission via medicalemployees and importance of routine infection-prevention policyin a nosocomial outbreak of Middle East respiratory syndrome (MERS): a descriptive analysis from a tertiary care hospital in South Korea. BMC Pulm Med 2019;19: 190. 